\title{
Vortex dynamics of clapping plates
}

\author{
Daegyoum Kim $^{1}$, Fazle Hussain ${ }^{2}$ and Morteza Gharib ${ }^{1} \uparrow$ \\ ${ }^{1}$ Division of Engineering and Applied Science, California Institute of Technology, \\ Pasadena, CA 91125, USA \\ ${ }^{2}$ Department of Mechanical Engineering, University of Houston, Houston, TX 77204, USA
}

(Received 16 November 2011; revised 18 August 2012; accepted 6 September 2012)

\begin{abstract}
Vortex formation and force generation of clapping plates with various aspect ratios $(A R)$ and stroke angles were investigated. Experiments were performed with a pair of hinged rectangular plates that were rotated symmetrically in a static fluid, and defocusing digital particle image velocimetry was employed to measure the threedimensional flow field. Single-plate cases were also studied to compare with clapping plate cases. As $A R$ decreases, both circulation of the tip vortex and area enclosed by the vortex loop increase inversely. An empirical power-law relationship with a negative exponent is found between total impulse and $A R$ for a given stroke angle. The sensitivity of the force generated by the plates to the change of $A R$ is larger at the smaller stroke angle because of faster acceleration and deceleration. The increase in impulse per plate from the single-plate case to the clapping double-plate case is larger for lower $A R$. These results reveal that low $A R$ wings are more efficient in propulsive force generation in some specific modes of unsteady flapping flight. The evolution of the wake structures is found to depend on $A R$ and stroke angle.
\end{abstract}

Key words: biological fluid dynamics, propulsion, swimming/flying, vortex dynamics, vortex flows

\section{Introduction}

Some flying animals propel themselves using a pair of symmetric wings that closely approach or touch each other. The most well-known case is the Weis-Fogh clap and fling mechanism used by hovering insects (Weis-Fogh 1973; Ellington 1984a). In this mechanism, a pair of wings clap and fling sequentially during pronation. Some insects such as locusts and butterflies clap and fling their wings even during forward flight (Cooter \& Baker 1977; Brodsky 1991). Several aquatic animals also press a pair of propulsors against each other for locomotion. For example, frogs kick their legs towards a sagittal plane when they move in water. A similar motion is also found when humans swim using the breaststroke. Some advantages of this locomotion mode have been proposed. For example, insect wings can generate large lift instantaneously when the wings fling apart after clapping; the sudden growth of the vortices over the wings has been regarded as an important unsteady lift enhancement mechanism in a clap and fling mode (Weis-Fogh 1973; Ellington 1984b). Gal \& Blake (1988) suggested that frogs generate additional thrust during extension of their legs 
where momentum is imparted to the surrounding water as a jet between the two legs. Brodsky (1991) claimed that butterflies use this jetting mechanism for thrust generation during the pronation phase.

Vortex dynamics has been studied to understand the relation between kinematics and propulsive force production for a pair of propulsors approaching or contacting each other. In the case of hovering insects, large leading-edge vortices were observed on the upper surfaces of flinging wings during pronation (Spedding \& Maxworthy 1986; Sun \& Yu 2003; Miller \& Peskin 2005). These vortices remain stable over the wings during the downstroke (Maxworthy 1979). Using smoke visualization, Brodsky (1991) showed that a vertical vortex ring was generated when butterfly wings clapped after the upstroke and that this vortex ring is connected to another vortex ring created during the downstroke. Using planar particle image velocimetry, Johansson \& Lauder (2004) and Stamhuis \& Nauwelaerts (2005) showed that vortices were shed off each leg when the frog kicked its legs and that these vortices remained separated from each other downstream. However, in all these cases, it was difficult to investigate the complicated three-dimensional vortex formation process in detail because of the limitation of the visualization techniques used. If a pair of propulsors approaches each other closely, the vortices generated by them interact with each other and develop a complicated three-dimensional form, often involving reconnection. Therefore, the understanding of vortex dynamics in clapping propulsion requires analysis of the three-dimensional flow field.

Motivated by a butterfly's stroke followed by clapping for thrust generation (Brodsky 1991), we have studied the vortex dynamics of clapping plates. The main purpose is to investigate the effect of the geometric and kinematic conditions such as aspect ratio $(A R)$ and stroke angle on force generation and vortex formation. Butterflies have relatively low $A R$ wings compared to other flying animals. Thus, we considered $A R$ of the plate as an important parameter in force generation. To simulate a stroke, two thin rectangular plates were rotated symmetrically with a $90^{\circ}$ angle of attack in a static fluid, and only one stroke was considered. We used the simplified kinematics for the model and neglected the effects of other factors such as repeated strokes, background flow and complex three-dimensional motion. We also examined single-plate cases by removing one plate in order to compare the single-plate and double-plate cases. Defocusing digital particle image velocimetry (DDPIV) (Willert \& Gharib 1992; Pereira \& Gharib 2002; Pereira et al. 2006) was used to study the three-dimensional flow fields, and a load cell was used for force measurements.

This paper is organized as follows. The experimental set-up and data analysis are described in $\S 2$. In $\S 3.1$, the results on thrust generation and vortex formation of a single rotating plate are presented. Single-plate and double-plate cases are compared in $\S 3.2$. In $\S 3.3$, the vortex dynamics in the wake is analysed for the double-plate case. Finally, results are summarized in $\S 4$.

\section{Experimental set-up}

Experiments were performed in a $103 \mathrm{~cm} \times 75 \mathrm{~cm} \times 82 \mathrm{~cm}$ glass tank filled with water (figure $1 a$ ). The plate actuation was controlled via a pair of stepper motors (NEMA 8, Anaheim Automation Inc.) mounted onto a traverse (Velmex Inc.) outside the tank. The plates were attached vertically to the end of the shafts. The $2 \mathrm{~cm}$ gap between the shafts rotating the plates was blocked using tape. The plates were $1.46 \mathrm{~mm}$ thick. The span $s$ of all plates was $8 \mathrm{~cm}$, and the chord $c$ ranged from 3 to $12 \mathrm{~cm}$ in $1 \mathrm{~cm}$ increments; the aspect ratio $A R(=s / c)$ ranged from 0.67 to 2.67 . For 
(a)

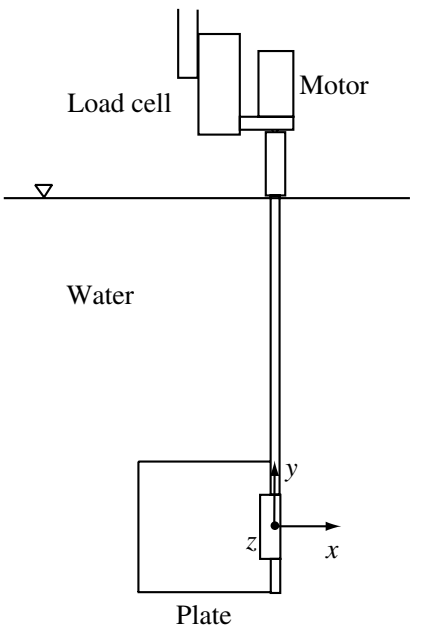

(b)
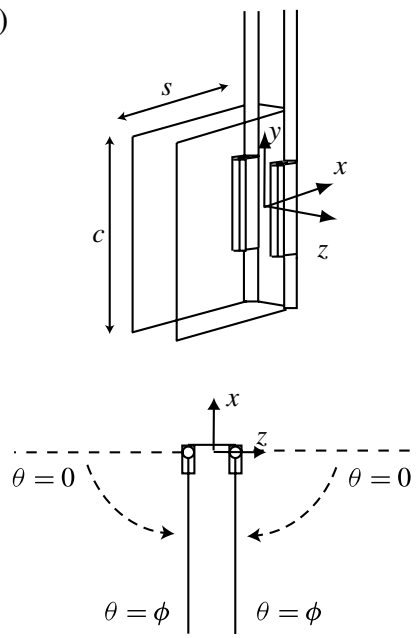

FIGURE 1. (a) Details of the model from the camera's view. (b) Definition of chord $c$, span $s$ and stroke angle $\phi$. The stroke angle $\phi$ is the same as the initial angle between the plate and the $z=0$ plane. The dashed arrows indicate the rotating direction of the plates. For the cases of single-plate configuration, the plate and shaft on the negative $z$-side were removed.

most butterfly species, the aspect ratio of one side forewing and hindwing pair is in the range of 1-2 (Betts \& Wootton 1988; Dudley 1990). Note that we have defined aspect ratio for a single plate, not for double plates, in this paper. Therefore, the aspect ratio of the plate we studied is relevant to that of butterflies.

Each plate was rotated with a sinusoidal angular velocity that varied with stroke angle $\phi$ (figure $1 b$ ) as

$$
\theta\left(t_{d}\right)=\frac{\phi}{T}\left(t_{d}-\frac{T}{2 \pi} \sin \left(\frac{2 \pi t_{d}}{T}\right)\right) \quad \text { and } \quad \dot{\theta}\left(t_{d}\right)=\frac{\phi}{T}\left(1-\cos \left(\frac{2 \pi t_{d}}{T}\right)\right),
$$

where $t_{d}$ is the dimensional time and $T$ is the rotation time. The plate was stopped at $t_{d}=T(\theta=\phi)$, parallel to the other plate; only one power stroke was considered. The initial position of the plate depended on the stroke angle $\phi$. The rotation time $T$ was proportional to the stroke angle $\phi: 1.2 \mathrm{~s}$ for $\phi=90^{\circ}$, and $2.4 \mathrm{~s}$ for $\phi=180^{\circ}$. The maximum and mean angular velocities were independent of the stroke angle. For all cases, the Reynolds number, $R e=U s / \nu$, was 8400 , where $U$ is the mean speed of the plate tip $\left(105 \mathrm{~mm} \mathrm{~s}^{-1}\right), s$ is the span and $v$ is the kinematic viscosity of water. The origin of the coordinate system is between the two shafts, at the mid-height of the plates (figure 1). The vertical $y$-axis corresponds to the body axis of the butterfly during clapping (Brodsky 1991) and the thrust that the model generates during clapping is in the positive $x$-direction. In this study, free stream or forward motion of the model was not considered.

A DDPIV camera composed of three charge-coupled device (CCD) sensors was placed outside the tank, with the camera probe volume in the middle of the tank. The water was seeded with silver-coated ceramic spheres of mean diameter $100 \mu \mathrm{m}$ (Conduct-o-fil, Potters Industries Inc.). An Nd:YAG laser (Gemini PIV, New Wave Research Inc.; $200 \mathrm{~mJ}$ pulse ${ }^{-1}$ ) was fired upwards from the bottom of the tank. Image pairs were recorded in a double-exposure mode with a time gap of $5 \mathrm{~ms}$ between laser pulses. The images from the DDPIV camera were processed with in-house 
DDPIV software based on Pereira \& Gharib (2002) and Pereira et al. (2006). The three-dimensional coordinates of the particles inside the tank were found by matching particle images of the three CCDs. Velocity vectors calculated by a three-dimensional particle tracking scheme (Pereira et al. 2006) were randomly spaced in a measurement volume of $160 \mathrm{~mm} \times 160 \mathrm{~mm} \times 160 \mathrm{~mm}$. The full flow field was divided into cubic grids of $3 \mathrm{~mm} \times 3 \mathrm{~mm} \times 3 \mathrm{~mm}$. Compared to the plate span $(s=80 \mathrm{~mm})$, the grid resolution of the vector field was fine enough to identify the vortex formation process. The number of randomly spaced velocity vectors obtained by particle tracking was $\sim 5 \times 10^{3}$ in a single experiment, and this was much smaller than the number of cubic grids in the mapped domain. With only a single experiment, it was difficult to map a reasonable velocity field interpolated on cubic grids. Therefore, to increase the number of randomly spaced velocity vectors, each experiment was repeated 30 times under the same conditions with a $60 \mathrm{~s}$ break between each run. We confirmed that $60 \mathrm{~s}$ was enough for the flow to settle down, by inspecting particle motion and processing PIV images. The randomly spaced velocity vectors were collected from all 30 runs and interpolated onto cubic grids with a $50 \%$ overlap to obtain a velocity field. After removing outliers and applying a smoothing operator to velocity fields, vorticity fields were obtained by a central difference scheme and then further smoothed. To estimate the variation of velocity field outputs by repeated runs, two variables, $\Gamma$ and $\boldsymbol{x}_{c}$ defined in (2.3), were compared among three sets of 10 runs for the $A R=1$ cases. The root mean-square (r.m.s.) deviations of $\Gamma$ and $\boldsymbol{x}_{c}$ among the three sets were within 8 and $3 \%$ of the mean values, respectively.

First, the flow structure around the rotating plates was studied for $A R=$ $0.67,0.8,1,1.33$ and 2 . Then, the vortex structure moving in the wake of the stopped plates was studied for $A R=0.67,1$ and 2 . In the $A R=0.67$ and 0.8 cases, the entire vortex structure could not be included in the mapped flow field as the plate was long along the $y$-direction for these two cases $(s=12$ and $10 \mathrm{~cm})$. For DDPIV measurement, we moved up the plate $4 \mathrm{~cm}$ for the $A R=0.67$ case and $2 \mathrm{~cm}$ for the $A R=0.8$ case along the $y$-axis to identify only the lower half of the vortex. The flow field was then mirrored across the symmetrical $y=0$ plane to obtain an entire vortex structure. Flow fields were measured for three stroke angles, $\phi=45,90$ and $135^{\circ}$. In some cases, the camera probe volume was not large enough to include the entire vortex structure in the $x$-direction. In these cases, two sets of the experiments were performed by taking measurements at an additional position downstream of the plates. For the second set, the model was translated in the $x$-direction. The velocity vectors of the two sets with different model positions were combined before interpolating to cubic grids. The total fluid volume mapped was $280(240) \mathrm{mm} \times 160 \mathrm{~mm} \times 160 \mathrm{~mm}$.

Time, position, velocity and vorticity were non-dimensionalized as

$$
t=t_{d} U / s, \quad \boldsymbol{x}=\boldsymbol{x}_{d} / s, \quad \boldsymbol{u}=\boldsymbol{u}_{d} / U, \quad \boldsymbol{\omega}=\boldsymbol{\omega}_{d} s / U,
$$

where $U$ and $s$ are the same for all cases. The non-dimensional circulation of the tip vortex $\Gamma$ and the non-dimensional position of the tip vortex centre $\boldsymbol{x}_{c}$ on the $y=0$ plane were calculated from the vorticity field as

$$
\Gamma=\int \omega_{y} \mathrm{~d} A \text { and } \boldsymbol{x}_{c}=\frac{\int \boldsymbol{x} \omega_{y} \mathrm{~d} A}{\Gamma},
$$

where $A$ is the positive $z$-domain on the $y=0$ plane. Vorticity shed from the tip of the plate, positive $\omega_{y}$, was used in the calculation of $\Gamma$ and $\boldsymbol{x}_{c}$. Any $\omega_{y}$ less than $3 \%$ of maximum $\omega_{y}$ on the plane $\left(\omega_{y}<0.03 \omega_{y}^{\max }\right)$ was discarded to avoid including noise. 
In addition, $0.02 \omega_{y}^{\max }$ and $0.04 \omega_{y}^{\max }$ were also considered to check the sensitivity of $\Gamma$ and $\boldsymbol{x}_{c}$ to the change of the threshold value. The maximum difference of $\Gamma$ and $\boldsymbol{x}_{c}$ between the $0.03 \omega_{y}^{\max }$ case and the other two cases was $5 \%$.

To measure the impulse generated by a rotating plate, a load cell (MB Miniature Beam, Interface Inc.) was attached to the motor. The plate stroke angle was varied from $\phi=45^{\circ}$ to $\phi=180^{\circ}$ in $45^{\circ}$ increments. The angular velocity of the plate followed (2.1). The signal was amplified and low-pass-filtered at $5 \mathrm{~Hz}$ by a signal conditioner (SGA, Interface Inc.). A thrust coefficient $C_{T}$ and a non-dimensional total impulse $I_{\infty}$ were computed as follows:

$$
C_{T}\left(t_{d}\right)=\frac{F_{x}\left(t_{d}\right)}{\frac{1}{2} \rho U^{2} \hat{r}_{2}^{2} S} \quad \text { and } \quad I_{\infty}=\frac{\int_{0}^{\infty} C_{T}\left(t_{d}\right) \mathrm{d} t_{d}}{T} .
$$

Here $F_{x}$ is the force acting on the model in the $x$-direction, $U$ is the mean tip velocity of the plate, $S$ is the area of the plate ( $S$ for the double plates is twice that of the single plate) and $\hat{r}_{2}^{2}=\int_{0}^{1}(r / s)^{2} \mathrm{~d}(r / s)$ is the non-dimensional second moment of plate area with respect to the rotating axis, where $r$ is a radial coordinate from the rotating axis to the plate tip. Note that the total impulse $I_{\infty}$ is divided by the rotation time $T ; I_{\infty}$ is different from the mean thrust coefficient during the rotation time,

$\bar{C}_{T}=\int_{0}^{T} C_{T}\left(t_{d}\right) \mathrm{d} t_{d} / T$, in that $I_{\infty}$ is integrated over infinite time. In some cases, when the plates stopped, $C_{T}$ had a negative value even though it soon returned to zero. In this sense, $I_{\infty}$ may be a better parameter than $\bar{C}_{T}$ in characterizing total force. The difference between $I_{\infty}$ and $\bar{C}_{T}$ was within $5 \%$ in most cases. Long after the plate stops, the voltage output of the load cell should return to its initial value. However, the voltage output did not return exactly to its initial value and was slightly off. In (2.4), the force should be integrated over infinite time to obtain $I_{\infty}$. Before $T+0.4 \mathrm{~s}$, $C_{T}$ returned to within \pm 0.1 of zero value. Therefore, the force was actually integrated from $t_{d}=0$ to $t_{d}=T+0.4 \mathrm{~s}$ to obtain $I_{\infty}$. We also measured force with the model in air and subtracted it from the force obtained in water, in order to neglect the inertial force component in the measured force. The force was averaged from six repeated measurements. The standard deviation of $I_{\infty}$ for six repeated measurements was within $6 \%$ of the mean value.

\section{Results and discussion}

\subsection{Vortex formation and force generation of a single plate}

For the single-plate configuration, only the plate on the positive $z$ side was attached to the apparatus. Figure 2 shows the instantaneous thrust coefficient $C_{T}$ for three $A R$ values (0.67, 1 and 2). During rotation, a lower $A R$ plate with longer chord $c$ generally produced larger $C_{T}$; dimensional thrust $F_{x}$, the numerator in the first equation of (2.4), is not simply proportional to the chord length or area of the plate. This inverse relation between $C_{T}$ and $A R$ was found at all stroke angles. The effect of $A R$ on force generation is more clearly shown in figure 3(a) for the four stroke angles $\left(\phi=45,90,135\right.$ and $\left.180^{\circ}\right)$. As does $C_{T}, I_{\infty}$ tends to decrease as $A R$ increases from 0.67 to 2.67. The experimental data in figure 3(a) were fitted by an empirical power law of the form $\alpha A R^{\beta}$ for each stroke angle. The exponent $\beta$ is -0.44 for $\phi=45^{\circ}$, -0.31 for $\phi=90^{\circ},-0.24$ for $\phi=135^{\circ}$ and -0.20 for $\phi=180^{\circ}$. Thus, in the range $\phi=45-180^{\circ}$, the absolute magnitude of the exponent decreases with stroke angle. This power law may not be valid beyond the $A R$ range considered here. Note that, as $A R$ 

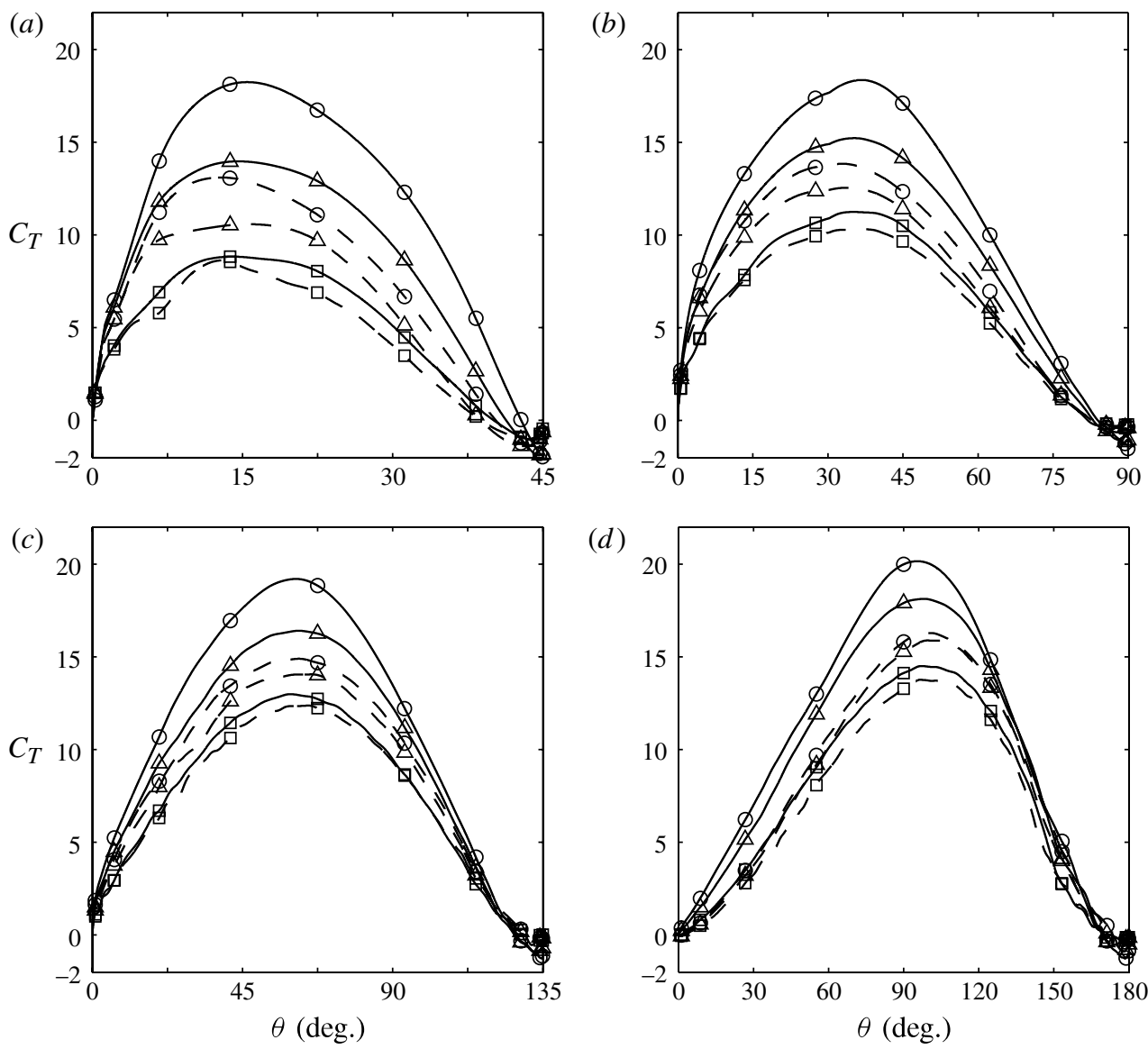

FIGURE 2. Instantaneous thrust coefficient $C_{T}$ as a function of rotating angle $\theta\left(t_{d}\right)$ defined in (2.1) at four stroke angles: $(a) \phi=45^{\circ} ;(b) \phi=90^{\circ} ;(c) \phi=135^{\circ}$; and $(d) \phi=180^{\circ}$. Symbols/curves: $\bigcirc, A R=0.67 ; \triangle, A R=1 ; \square, A R=2 ;--$, single plate; and - , double plates.

decreases to zero (chord length increases infinitely), the flow becomes two-dimensional and $I_{\infty}$ should approach a finite value, which demonstrates the limit of the empirical power-law fitting.

We examine the dependence of $I_{\infty}$ on $A R$ with a basic blade element theory that considers quasi-steady force and added-mass reaction force. The force generation during unsteady translating or rotating motion of the plate with a $90^{\circ}$ angle of attack cannot be explained simply by the blade element theory. Moreover, the blade element approach, using chordwise strips along the span, is available only when $A R$ is large enough to assume that the flow is two-dimensional at each chordwise strip. However, this analytical approach provides useful information to understand the relation between $A R$ and thrust generation of our model. In the blade element approach, for a chordwise strip at a given radial distance from the rotating axis, while the quasi-steady force normal to the chord is proportional to the chord, the added-mass force is proportional to the chord squared. Therefore, the contribution of the quasi-steady force component to $I_{\infty}$ or $C_{T}$ is independent of $A R$, but that of the added-mass force component is 

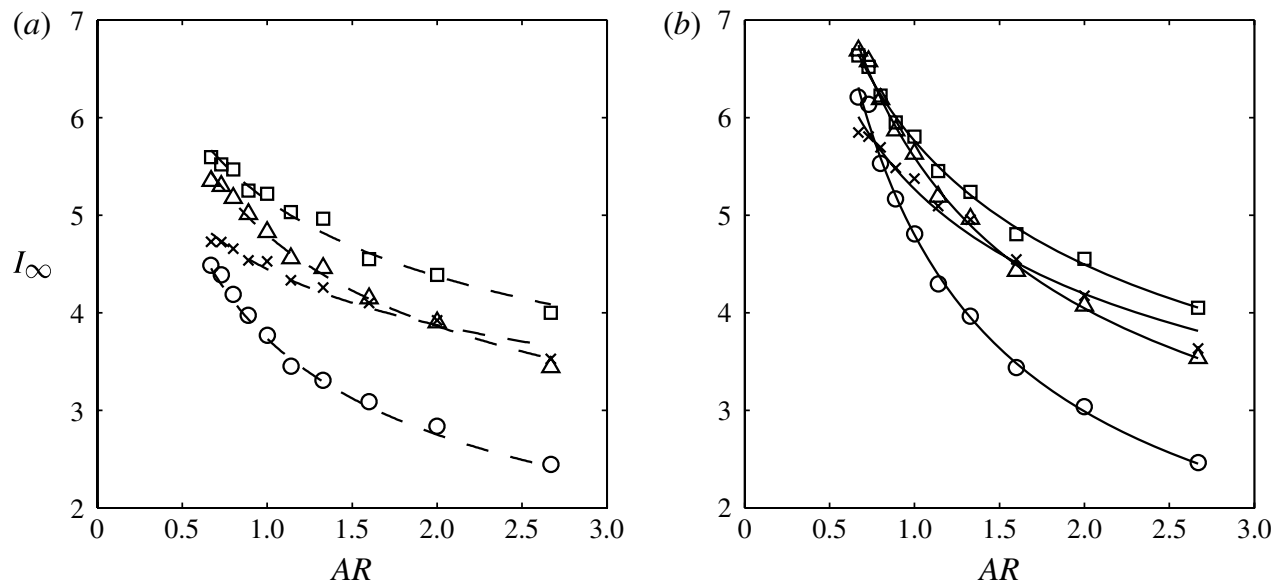

FIGURE 3. Total impulse $I_{\infty}$ for $(a)$ single plate and $(b)$ double plates, in the range $A R=0.67-2.67$, at four stroke angles: $\bigcirc, 45^{\circ} ; \triangle, 90^{\circ} ; \square, 135^{\circ}$; and $\times, 180^{\circ}$.

(a)

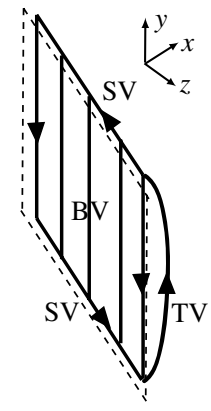

(b)

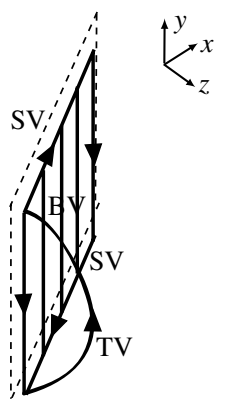

(c)

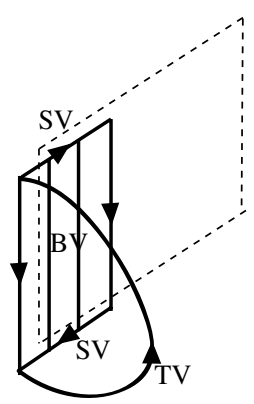

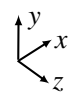

FIGURE 4. Simplified vortex line representation of sequential vortex formation process for a single rotating plate: TV, (starting) tip vortex; SV, side vortex; and BV, bound vortex. Arrows indicate the rotating direction of the vortex using the right-hand rule, and dashed lines are the edges of the plate. The plate rotates with respect to the negative $y$-axis.

inversely proportional to $A R$. Thus, if the exponent $\beta$ is closer to -1 rather than 0 in the power-law relation between $A R$ and $I_{\infty}$, it implies that the contribution of the unsteady force to $I_{\infty}$ becomes larger than that of the quasi-steady force. In figure $3(a)$, the negative exponent in the power law is mainly due to unsteady force generation by sinusoidal rotation. For the stroke angles studied here, the $\phi=45^{\circ}$ case whose plate motion is the most unsteady, with fast acceleration and deceleration, has the largest absolute magnitude of the exponent.

Before discussion of vortex formation, some terms are clarified (figure 4). As a plate rotates, a (starting) tip vortex and side-edge vortices develop at a vertical edge, and at upper and lower horizontal edges of the plate, respectively. A bound vortex is the collection of vorticity close to the surface of the plate and is the counterpart of the starting tip vortex. The bound vortex and the starting tip vortex have $\omega_{y}$ of opposite signs. The boundary layer of the plate is a major part of the bound vortex. A closed 

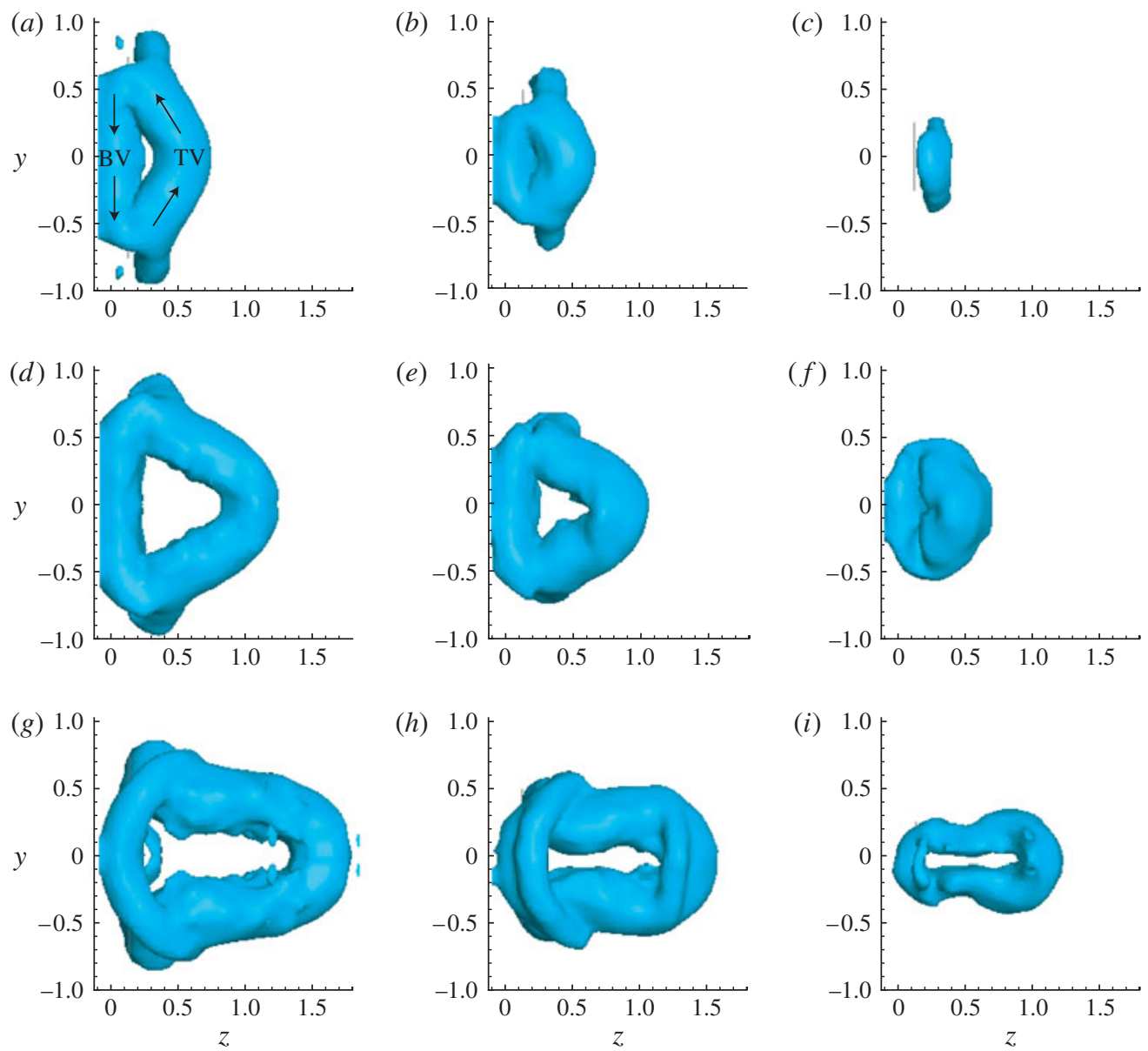

FIgURE 5. (Colour online) Vortex structure from the back view (from $-x$ ) when a single plate stops: (a) $\phi=45^{\circ}, A R=0.67$; (b) $\phi=45^{\circ}, A R=1 ;$ (c) $\phi=45^{\circ}, A R=2$; (d) $\phi=90^{\circ}, A R=0.67$; (e) $\phi=90^{\circ}, A R=1 ;(f) \phi=90^{\circ}, A R=2 ;(g) \phi=135^{\circ}, A R=0.67$; (h) $\phi=135^{\circ}, A R=1$; (i) $\phi=135^{\circ}, A R=2$. The isosurfaces represent $|\omega|=4$. The plate is positioned vertically at $z=0.125$. TV, (starting) tip vortex; and $\mathrm{BV}$, bound vortex.

vortex loop is formed from the shedding tip vortex and bound vortex after the plate stops.

When a plate starts to rotate, a (starting) tip vortex is rolled up along the vertical tip edge of the plate. However, the tip vortex begins to separate from the tip edge near the symmetrical $y=0$ plane and becomes curved. While the plate continues to rotate, the tip vortex moves farther away from the plate (figure 4). More details on the tip vortex dynamics of a rotating plate with a $90^{\circ}$ angle of attack were described in Kim \& Gharib (2011). For a given stroke angle, the extent of tip vortex separation is influenced by $A R$. In figure 5, the vortex structures are plotted for three different $A R$ values $(0.67,1$ and 2$)$ when plate motion stops. One noticeable difference among the three $A R$ cases of the same stroke angle is the position of the tip vortex in the $z$-direction. For the lower $A R$ cases, the tip vortex is positioned farther from the tip edge of the plate along the $z$-direction (figure $6 a$ ). For example, in the $\phi=90^{\circ}$ case, 

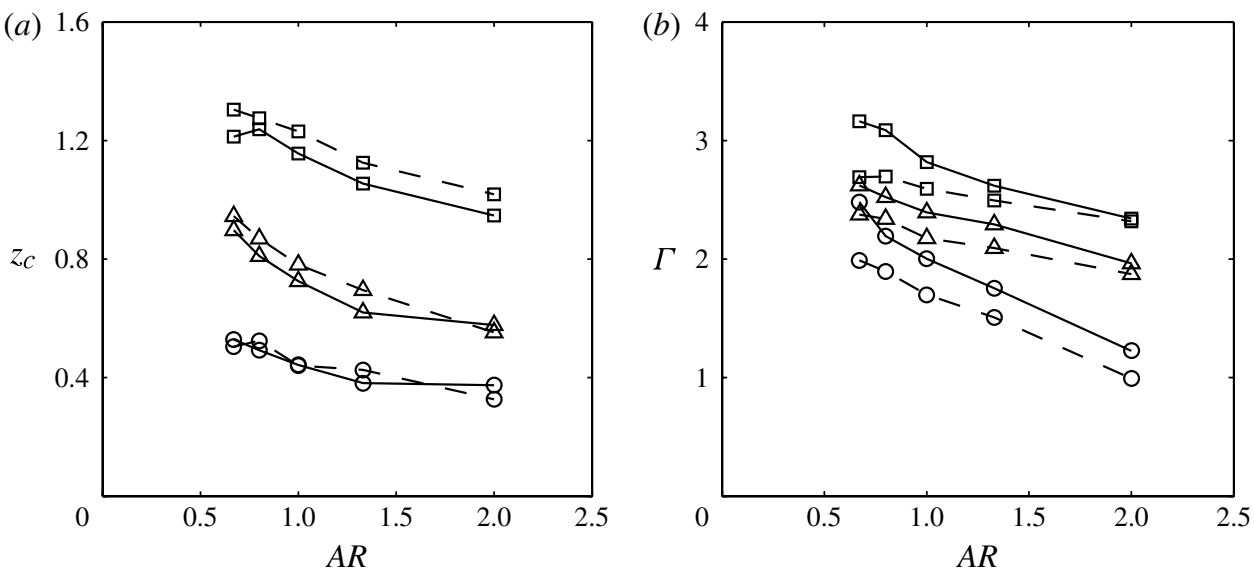

FIGURE 6. (a) Location of the vortex centre in the $z$-direction and $(b)$ circulation of the tip vortex in the $A R=0.67-2$ range. Symbols/curves: -- , single plate; - , double plates; $\bigcirc$, $\phi=45^{\circ} ; \triangle, \phi=90^{\circ}$; and $\square, \phi=135^{\circ}$.

the centre of the tip vortex on the $y=0$ plane is at $z=1.0$ for $A R=0.67$, at $z=0.8$ for $A R=1$ and at $z=0.6$ for $A R=2$. As $A R$ decreases, separation of the tip vortex from the tip edge occurs earlier during rotation. This separation process results in a larger area of the loop enclosed by the starting tip vortex and the bound vortex. The $A R$ dependence of the tip vortex separation is attributed to side-edge vortices. Flow induced by two side-edge vortices with opposite rotations induces the tip vortex to follow the tip edge (figure 4). However, when the distance between the two side-edge vortices is large, their influence on the motion of the tip vortex will be diminished, especially near the $y=0$ plane. In addition, near the plate corners, the vortex has a high curvature due to the $90^{\circ}$ corner. Thus, the self-induced velocity of the vortex near the corner region is high in the direction bi-normal to the tip edge and the side edge. Thus, the vortex near the corner can follow the plate motion. However, the tip vortex near the $y=0$ plane is less affected by the self-induced bi-normal motion of the vortex near the corner. For these reasons, the tip vortex of the plate with a longer chord will undergo earlier separation.

The strength of the tip vortex continues to grow during rotation, and it is also affected by $A R$. During rotation, the plate with lower $A R$ generally has higher instantaneous circulation $\Gamma$ of the tip vortex, which is closely related with our result that the lower $A R$ plate has larger instantaneous thrust coefficient $C_{T}$. As a result, the circulation $\Gamma$ at the stopping time is in an inverse relation with $A R$ (figure $6 b$ ). The difference of $\Gamma$ between $A R=0.67$ and $A R=2$ is 1.0 for $\phi=45^{\circ}, 0.5$ for $\phi=90^{\circ}$ and 0.4 for $\phi=135^{\circ}$. The $A R=2$ and $\phi=45^{\circ}$ case shows a distinct decline of $\Gamma$ just before the plate stops: $\Gamma$ drops 0.3 during the last $1.3^{\circ}$ of rotation. Since the starting tip vortex is close to the tip edge in the $A R=2$ and $\phi=45^{\circ}$ case, when the plate decelerates, some vorticity of the bound vortex is shed into the starting tip vortex of opposite sign. Consequently, the starting tip vortex is annihilated. If the starting tip vortex and the bound vortex are more distant during deceleration, as in the $\phi=90$ and $135^{\circ}$ cases, the circulation of the tip vortex does not decline significantly during deceleration. 


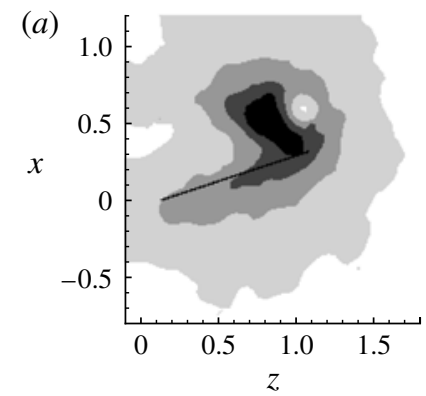

(b)

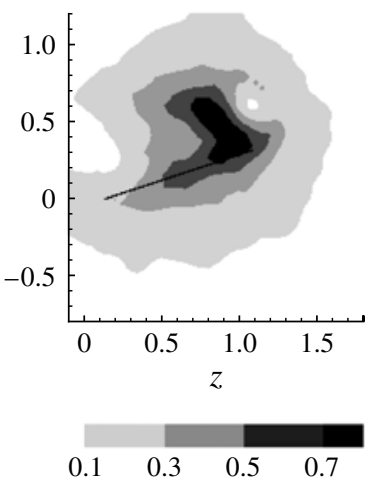

(c)

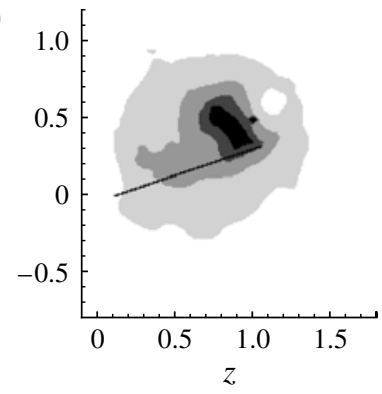

FIGURE 7. Distribution of velocity magnitude $|\boldsymbol{u}|$ on the $y=0$ plane at $\theta=26^{\circ}$ for the stroke-angle $\phi=135^{\circ}$ case: $(a) A R=0.67 ;(b) A R=1$; and $(c) A R=2$. The view is from $+y$. The plate is shown as a black line.

To explain why a plate with lower $A R$ generates larger circulation, the effect of side-edge vortices on tip vortex strength is first examined. The side-edge vortices induce a flow normal to the surface of the plate, decreasing the effective velocity of the plate and hence the circulation of the tip vortex. Here, the effective velocity is the apparent flow velocity encountered by the front (pressure) surface of the plate. As the chord decreases, the induced flow by the side-edge vortices becomes more dominant, and the circulation of the tip vortex decreases. However, this argument cannot explain why the circulation of larger $A R$ is always higher during the entire rotation time. When the plate begins to rotate, the side-edge vortex is not developed enough to affect the circulation growth of the tip vortex. Thus, another mechanism seems necessary to explain the $A R$ dependence of circulation.

Figure 7 shows the velocity distribution on the $y=0$ section for three $A R$ values with $\phi=135^{\circ}$ at an early stage of rotation $\left(\theta=26^{\circ}\right)$. For lower $A R$, more fluid is accelerated towards the back (suction) surface of the plate and away from the front surface at the $y=0$ section. This trend is generally applicable to other horizontal sections with the same $y / c$ in addition to the $y=0$ section. In other words, as the chord length increases, the volume of fluid in motion per chord length increases as well during the early stage of rotation. From the dynamic relation between the pressure gradient and acceleration of the fluid, it is expected that the pressure difference between the front and back surfaces of the plate is larger for lower $A R$ (longer chord), especially when the side-edge vortex size is not large enough to affect significantly the flow behind the plate. The pressure difference between the front and back surfaces of the plate causes the growth of the tip vortex. Therefore, for lower $A R$, the tip vortex strength becomes larger during the early stage of rotation.

The force acting on a very thin body in incompressible and viscous flow is $\boldsymbol{F}\left(t_{d}\right)=-\mathrm{d} \boldsymbol{P} / \mathrm{d} t_{d}$, where $\boldsymbol{P}$ is fluid impulse, $\boldsymbol{P}=\rho \int_{V_{\infty}} \boldsymbol{x}_{d} \times \boldsymbol{\omega}_{d} \mathrm{~d} V_{d} / 2$, and $V_{\infty}$ is an infinite fluid domain at rest at infinity ( $\mathrm{Wu} 1981)$. Therefore, the dimensional impulse acting on the plate $\left(=\int_{0}^{\infty} F_{x}\left(t_{d}\right) \mathrm{d} t_{d}\right)$ is the $x$-component of $\boldsymbol{P}$. In nondimensionalization with (2.2) and (2.4), the impulse $I_{\infty}$ is as follows:

$$
I_{\infty}=\frac{s}{\hat{r}_{2}^{2} c T} \int_{V_{\infty}}\left(-y \omega_{z}+z \omega_{y}\right) \mathrm{d} V
$$




\begin{tabular}{lcccc}
$A R$ & \multicolumn{4}{c}{$\phi$} \\
& $45^{\circ}$ & $90^{\circ}$ & $135^{\circ}$ & $180^{\circ}$ \\
0.67 & $1.73(1.39)$ & $1.34(1.25)$ & $1.04(1.19)$ & $1.12(1.24)$ \\
1 & $1.04(1.28)$ & $0.80(1.17)$ & $0.59(1.11)$ & $0.85(1.19)$ \\
2 & $0.20(1.07)$ & $0.17(1.04)$ & $0.16(1.04)$ & $0.25(1.06)$
\end{tabular}

TABLE 1. Values of $\Delta I_{\infty}$, the increase in total impulse from single plate to double plates, for four stroke angles $\phi$ and three $A R$ values. The numbers in parentheses are the ratios of two total impulses, $I_{\infty}^{d} / I_{\infty}^{s}$.

\begin{tabular}{lrrrr}
$\phi$ & \multicolumn{1}{c}{$45^{\circ}$} & \multicolumn{1}{c}{$90^{\circ}$} & \multicolumn{1}{c}{$135^{\circ}$} & \multicolumn{1}{c}{$180^{\circ}$} \\
$\beta_{s}$ & -0.44 & -0.31 & -0.24 & -0.20 \\
$\beta_{d}$ & -0.68 & -0.47 & -0.36 & -0.33 \\
$\Delta|\beta|$ & 0.24 & 0.16 & 0.12 & 0.13
\end{tabular}

TABLE 2. Comparison of power-law exponents for single plate $\left(\beta_{s}\right)$ and double plates $\left(\beta_{d}\right)$; $\Delta|\beta|$ is $\left|\beta_{d}\right|-\left|\beta_{s}\right|$.

This equation shows the relationship between vorticity distribution $\left(y \omega_{z}\right.$ and $\left.z \omega_{y}\right)$ and non-dimensional total impulse. We have shown above that the strength and shape of the tip vortex are influenced by $A R$. For low $A R$, the early separation of the tip vortex from the plate tip edge during rotation increases the $z$ moment of $\omega_{y}$ inside the tip vortex, $z \omega_{y}$. Moreover, the large circulation of the tip vortex also contributes to the increase in $z \omega_{y}$. These two characteristics of vortex formation are correlated with the larger $I_{\infty}$ for the lower $A R$ plates. Based on the study of a single plate, we investigate double-plate cases in the following section.

\subsection{Comparison of single plate and double plates}

In the clapping double plates, the thrust coefficient $C_{T}$ and total impulse $I_{\infty}$ increase from that of the single plate for every stroke angle and $A R$ combination (figures 2 and $3)$. Note that the number of plates was considered in the definition of $C_{T}$ and $I_{\infty}$ in (2.4). The change of $I_{\infty}$ from the single-plate to the double-plate case, $\Delta I_{\infty}=I_{\infty}^{d}-I_{\infty}^{s}$, is dependent on $A R ; \Delta I_{\infty}$ is generally larger for lower $A R$ (table 1). For instance, in $\phi=45-180^{\circ}, \Delta I_{\infty}$ ranges from 1.04 to 1.73 for $A R=0.67$. Meanwhile, $\Delta I_{\infty}$ ranges only from 0.16 to 0.25 for $A R=2$. For $A R=0.67$ and $1, \Delta I_{\infty}$ is the largest at $\phi=45^{\circ}$, and deceases gradually as the stroke angle increases up to $135^{\circ}$. Above $\phi=135^{\circ}, \Delta I_{\infty}$ increases slightly. The ratio of the total impulse, $I_{\infty}^{d} / I_{\infty}^{s}$, also shows trends similar to $\Delta I_{\infty}$. The magnitude of the exponent of the power-law fitting is also larger for the double plates in the range $\phi=45-180^{\circ}$ (figure 3 and table 2). The increase in the exponent magnitude from the single plate to the double plates, $\Delta|\beta|$, was at least 0.11 in this stroke angle range. This increase shows that thrust generation is more strongly affected by the variation of $A R$ in the double plates than in the single plate.

The double plates produce a tip vortex with a larger circulation than the single plate for $A R$ values between 0.67 and 2 (figure $6 b$ ). Like total impulse, the increase in circulation from the single plate to the double plates is generally larger for lower $A R$. However, the position of the tip vortex centre in the $z$-direction does not show any noticeable increase, but rather decreases for $\phi=90$ and $135^{\circ}$ (figure $6 a$ ). For the 

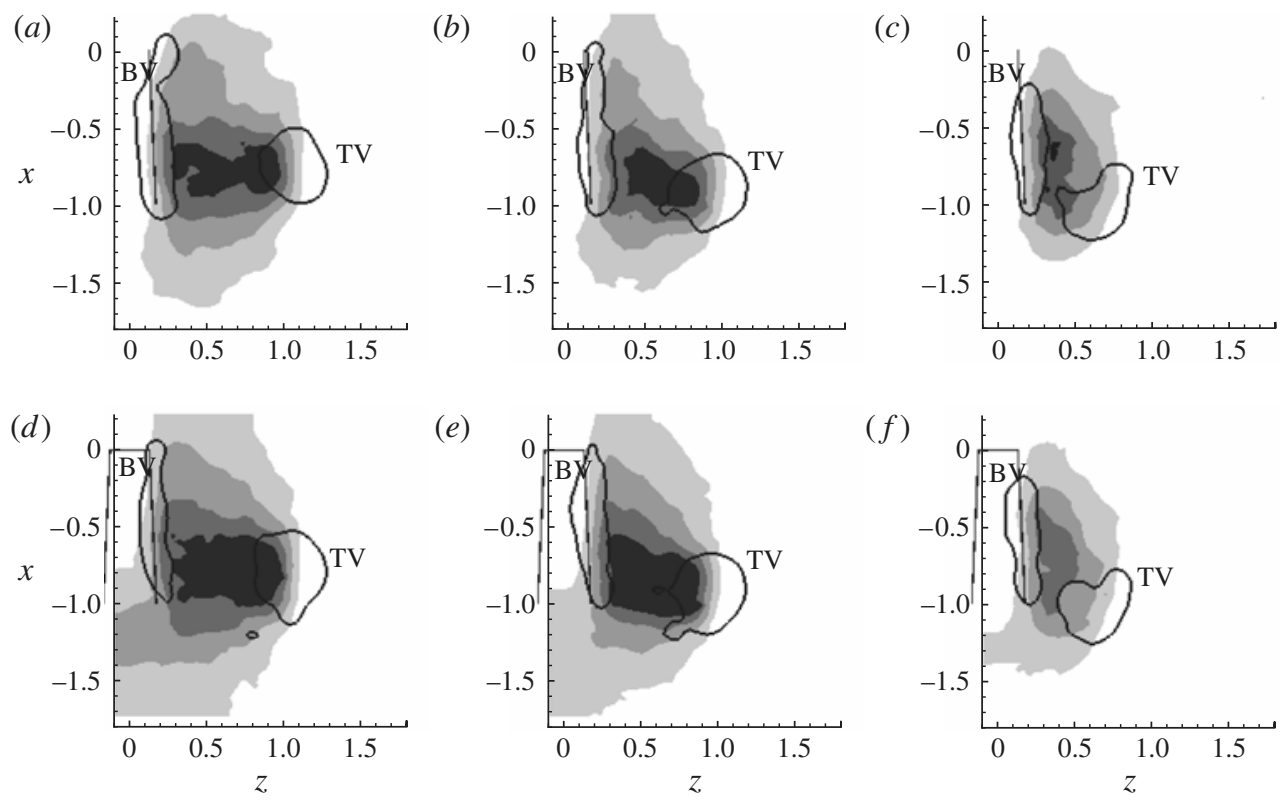

(e)

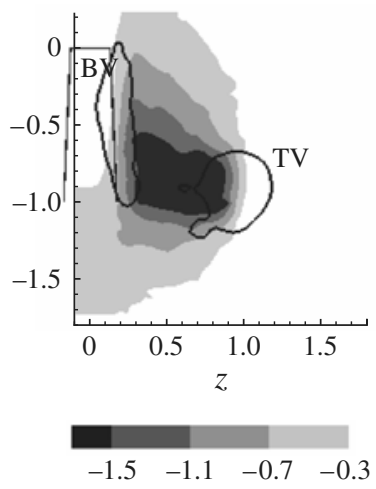

$(f)$

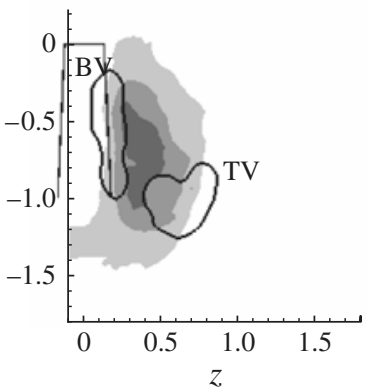

FIGURE 8. Distribution of the $x$-component of velocity $u_{x}$ on the $y=0$ plane at $\theta=87^{\circ}$ for the stroke-angle $\phi=90^{\circ}$ case: (a) $A R=0.67$, single plate; (b) $A R=1$, single plate; (c) $A R=2$, single plate; $(d) A R=0.67$, double plates; $(e) A R=1$, double plates; $(f) A R=2$, double plates. The view is from $+y$, and the downstream direction is $-x$. Since $z<-0.1$ was not mapped, the flow field of the left plate is not shown for the double-plate cases. Thin lines are plate edges, and thick lines are tip vortex and bound vortex.

clapping plates, the direction of the overall fluid momentum generated by the plates is parallel to the negative $x$-axis because of the symmetric motion of the plates with respect to the $z=0$ plane. Moreover, when two plates approach each other, the flow induced by a plate augments the effective velocity of the other plate. Thus, the tip vortex of one plate is strengthened by the flow generated by the other plate. On the other hand, the fluid between two closing plates can leak through the upper and lower gaps between the two plates instead of being entrained in the tip vortex or being expelled downstream. For high $A R$ plates with a short chord length, a larger proportion of the fluid between the two plates will leak through the upper and lower gaps than for low $A R$ plates. This can partially counteract the increase in the tip vortex strength and force generation. If the front surfaces of the two plates are initially close to each other, then each plate is under the strong influence of its counterpart during the whole time of rotation. Therefore, in spite of the shortest time of rotation, the $\phi=45^{\circ}$ cases have a circulation increase from the single plate to the double plates comparable to or even larger than that of any other stroke angle.

The distribution of the $x$-component of fluid momentum around the plates is another indication of total thrust acting on the plate. The distribution of $u_{x}$ on the $y=0$ plane is shown in figure 8 for the single plate $(a-c)$ and double plates $(d-f)$. In figure 8 , the plates are in a deceleration phase just before stopping. In the double-plate case, fluid is pushed out downstream by the clapping plates as a jet and has negative $x$-momentum 
(a)

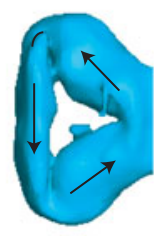

(b)

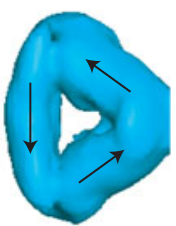

(c)

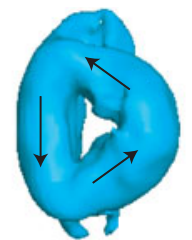

$(d)$

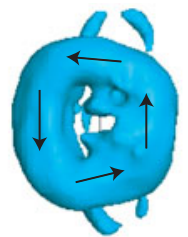

(e)

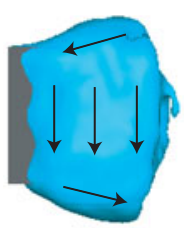

$(f)$

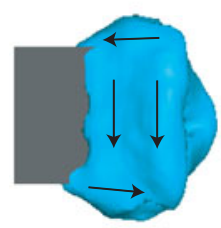

$(g)$

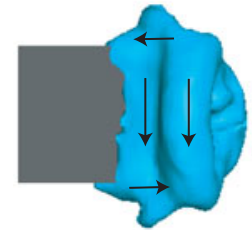

(h)

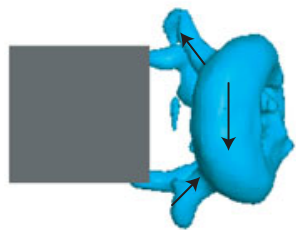

FIGURE 9. (Colour online) Vortex dynamics during $\tau=-0.26$ to 0.79 for the single plate with $\phi=90^{\circ}$ and $A R=1(|\omega|=4)$. $(a-d)$ Back view from $-x$ : $(a) \tau=-0.26$; $(b) \tau=0$; (c) $\tau=0.26$; (d) $\tau=0.79$. (e-h) Side view from $-z$ : (e) $\tau=-0.26 ;(f) \tau=0 ;(g) \tau=0.26$; (h) $\tau=0.79$. Arrows indicate the direction of rotation of the vortex.

near the symmetrical $z=0$, contrary to the single plate. The magnitude of $u_{x}$ near $z=0$ is larger in the lower $A R$ plate. This clearly demonstrates that, for the lower $A R$ plate, the fluid between the two plates tends to move in the negative $x$-direction rather than leaking in the $y$-direction through the upper and lower gaps of the plates. However, the region of highest fluid momentum is not near $z=0$, but between the counter-rotating tip vortex and the bound vortex on the same side. As expected from the relation between $A R$ and vortex shape and strength (figures 5 and 6), the magnitude of $u_{x}$ in this region also increases with decreasing $A R$. In the double-plate case, after the plates stop, two regions of high $u_{x}$ magnitude in both plates combine near $z=0$ and then move straight downstream; we will explain the vortex dynamics corresponding to this phenomenon in $\S 3.3$.

In our model, the non-dimensional total impulse and instantaneous thrust coefficient favour the lower $A R$ in both single plate and double plates. If the positive $x$-direction of our coordinate system is assumed to be the lift direction, the motion of our model is similar to the power stroke for hovering with a vertical stroke plane or the power stroke for very slow forward flight of flying animals. In these modes, a propulsor with lower $A R$ produces a larger lift coefficient according to the results presented above. The strength and shape of the tip vortex are important in propulsive force generation. Advance ratio is one of the parameters characterizing the unsteadiness of the flapping motion, and it is defined as the forward speed of the body divided by the mean flapping speed of the wing (Ellington 1984a). From this, we can infer that the effect of small $A R$ on lift generation may be reversed from positive to negative as the advance ratio of flight increases from zero (hovering) to infinity (gliding).

\subsection{Wake dynamics of clapping plates}

In the single plate, after the plate stops, the bound vortex rolls up into a circular stopping tip vortex and forms a vortex loop with the starting tip vortex (figure 9). From now on, another non-dimensional time variable $\tau\left(\tau=U\left(t_{d}-T\right) / s\right)$ will be used instead of $t\left(t=U t_{d} / s\right) ; \tau=0$ when the plate stops. Since $t$ at the stop depends on the stroke angle, we use $\tau$ to compare vortex dynamics of different stroke-angle cases. 
(a)

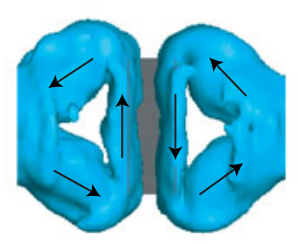

(b)

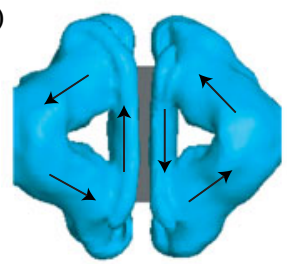

$(f)$

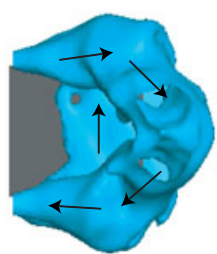

(c)

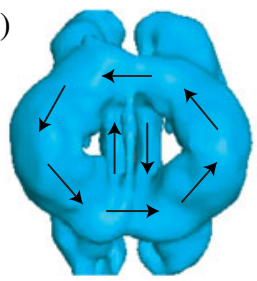

$(g)$

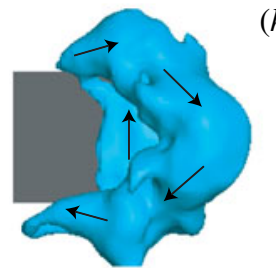

$(d)$

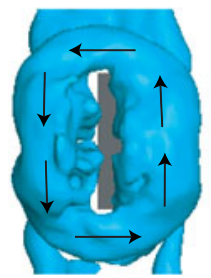

(h)

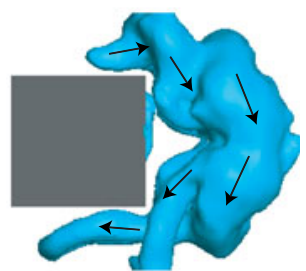

FIgURE 10. (Colour online) Vortex dynamics during $\tau=-0.26$ to 0.79 for the double plates with $\phi=90^{\circ}$ and $A R=1(|\omega|=4)$. $(a-d)$ Back view from $-x$ : $(a) \tau=-0.26$; $(b) \tau=0$; (c) $\tau=0.26$; $(d) \tau=0.79$. (e-h) Side view from $-z$ : $(e) \tau=-0.26 ;(f) \tau=0 ;(g) \tau=0.26$; (h) $\tau=0.79$. Arrows indicate the direction of rotation of the vortex.

(a)

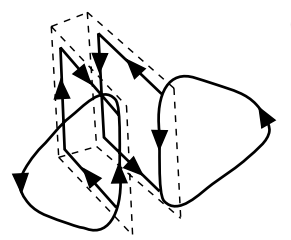

(b)

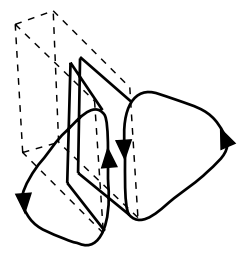

(c)

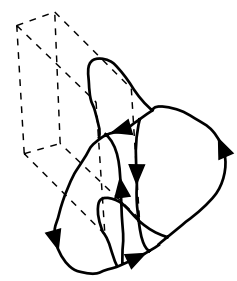

(d)

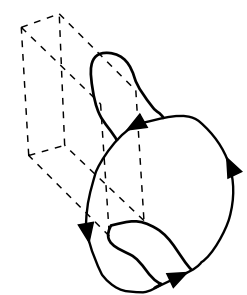

FIGURE 11. Simplified vortex line representation for the double plates with $\phi=90^{\circ}$ and $A R=1:(a) \tau=-0.26$; (b) $\tau=0$; (c) $\tau=0.26$; (d) $\tau=0.79$. Dashed lines are the edges of the plates.

Through mutual interaction of its counter-rotating parts, the vortex loop propagates downstream. Even though side-edge vortices and a portion of the bound vortex are also shed in the vertical direction from the plate, the vortex loop composed of the starting and stopping tip vortices is generally dominant in size and magnitude. The moving direction of the vortex loop is not parallel to the $x$-axis, but tilts in the negative $z$-axis.

Figures 10 and 11 show the dynamics of the wake structure for the clapping double plates $\left(A R=1\right.$ and $\left.\phi=90^{\circ}\right)$. At $\tau=0$, the shape of the tip vortex on each plate looks similar to that of the single plate in figure 9 . However, when the bound vortices on both plates begin to shed from the plates during the deceleration phase, they contact and cancel each other on the symmetrical $z=0$ plane. Simultaneously, the starting tip vortices in both plates connect near the upper and lower corners of the plates and form one vortex loop. The strength of the vortex loop at the connection continues to grow and saturates eventually. Not all of the bound vortex is involved in the cancellation process, but some thread remains behind the translating vortex loop. When two anti-parallel vortex tubes of similar strength collide in a viscous flow, they merge in a finite contact zone and cancel the vorticity of the vortex tube 

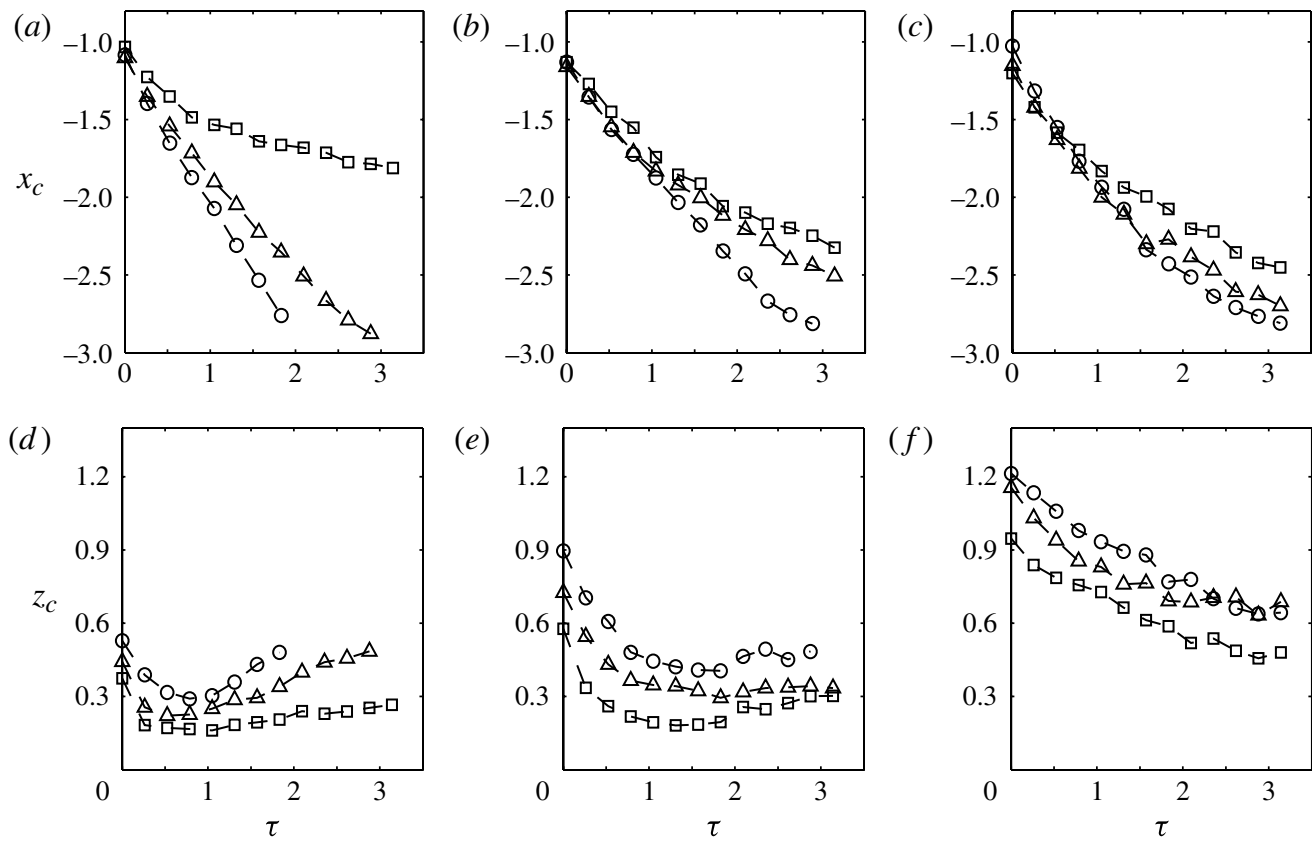

FIGURE 12. Location of the centre of the vortex loop in the wake in $(a-c)$ the $x$-direction and $(d-f)$ the $z$-direction: $(a) \phi=45^{\circ} ;(b) \phi=90^{\circ} ;(c) \phi=135^{\circ} ;(d) \phi=45^{\circ} ;(e) \phi=90^{\circ}$; ( $f) \phi=135^{\circ}$. Symbols/curves: $\bigcirc, A R=0.67 ; \triangle, A R=1$; and $\square, A R=2$.

on the opposite side. Bridges, vortex tubes perpendicular to the original vortex tubes, form near both ends of the contact zone and connect the two anti-parallel vortex tubes. This complicated phenomenon is known as the vortex reconnection mechanism (Melander \& Hussain 1989; Kida \& Takaoka 1994). Here, we have shown that vortex reconnection is important in the formation of the vortex loop from a pair of clapping plates. The side-edge vortices are still connected with the vortex loop when the loop moves downstream. Since the upper and lower side-edge vortices tend to move in the positive and negative $y$-directions, respectively, they are stretched by the vortex loop translating in the negative $x$-direction.

For the double plates, the evolution of the vortex loop in the wake was compared for three $A R$ values (2, 1 and 0.67) and three stroke angles (45, 90 and $\left.135^{\circ}\right)$. The translating speed of the vortex loop in the negative $x$-direction for the $\phi=45^{\circ}$ and $A R=2$ case is much lower than that of the other cases (figure $12 a-c$ ). In this case, the slow propagation of the vortex loop is because it becomes significantly weaker after $\tau=0$ due to the shedding of the bound vortex into the starting tip vortex on the same side. For $\phi=90$ and $135^{\circ}$, the difference in the downstream speed of the vortex loop among the three $A R$ values is not as noticeable as for $\phi=45^{\circ}$. The inner area enclosed by the vortex loop for $\phi=90$ and $135^{\circ}$ is larger than that of $\phi=45^{\circ}$. Therefore, for both $A R=0.67$ and 1, the propagating speed of the vortex loop by self-induction for $\phi=90$ and $135^{\circ}$ is smaller than that for $\phi=45^{\circ}$ in spite of the higher strength of the vortex loop.

For $\phi=45^{\circ}$ and $A R=1$, the starting tip vortices continue to move towards the symmetrical $z=0$ plane even after $\tau=0$. Therefore, the vortex loop compresses in the $z$-direction and elongates in the $y$-direction (figures $12 d$ and 13). The vortex loop 
(a)

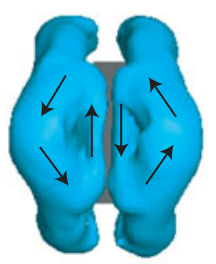

(b)

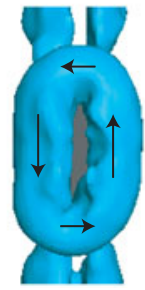

(c)

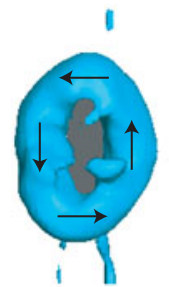

$(d)$

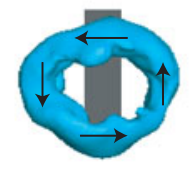

FIGURE 13. (Colour online) Vortex dynamics in the wake for the double plates with $\phi=45^{\circ}$ and $A R=1$ (back view from $-x,|\omega|=3.5$ ): (a) $\tau=0 ;$ (b) $\tau=0.79$; (c) $\tau=1.57$; (d) $\tau=2.36$.

is elliptical, with the major axis along the $y$-axis and the minor axis along the $z$-axis. The noticeable lateral compression of counter-rotating tip vortices within a short time causes the reduction of $z \omega_{y}$ in (3.1) and, consequently, causes the decrease of the total impulse after the plates stop. The total impulse $I_{\infty}\left(=\int_{0}^{\infty} C_{T}\left(t_{d}\right) \mathrm{d} t_{d} / T\right)$ was about $10 \%$ smaller than the mean thrust coefficient $\bar{C}_{T}$ integrated over the rotation time $T\left(=\int_{0}^{T} C_{T}\left(t_{d}\right) \mathrm{d} t_{d} / T\right)$. Similar morphodynamics of the vortex loop are also shown in the $\phi=45^{\circ}$ and $A R=0.67$ case. Even though the strength of the vortex loop is weakened by collision of the two tip vortices, they become so close to each other that the vortex loop can move downstream faster than that for $\phi=90$ or $135^{\circ}$. When the vortex loop travels downstream, it switches major and minor axes due to the non-uniform curvature of the vortex loop (figure 13b,d). Figure 12(d) also shows that, after reaching its minimum, the distance between the counter-rotating tip vortices on the $y=0$ plane increases for $A R=1$ and 0.67 . The axis-switching deformation of the vortex loop is analogous to that of an elliptical vortex ring generated from a nozzle exit (Kambe \& Takao 1971; Hussain \& Husain 1989). This axis-changing deformation occurs far downstream of the plate model and does not influence force generation of the model.

On the other hand, for all three $A R$ values with $\phi=90^{\circ}$, the vortex loop compresses laterally and elongates vertically after $\tau=0$ as well. However, unlike $\phi=45^{\circ}$, the switch of the major and minor axes in the vortex loop does not occur distinctly within the observed time (figure $12 e$ ). For $\phi=90^{\circ}$, the tip vortex is farther from the plate during rotation, compared to $\phi=45^{\circ}$, which implies that the tip vortex is less influenced by the plate motion. Moreover, the angular deceleration of the rotating plate takes twice as much time. Thus, the motion of the tip vortex towards the $z=0$ plane is slower than that for $\phi=45^{\circ}$. This results in a delayed lateral compression of the vortex loop. While the time when the two tip vortices are closest on the $z=0$ plane is between $\tau=0.5$ and 0.8 for $\phi=45^{\circ}$, it is between $\tau=1.3$ and 1.8 for $\phi=90^{\circ}$. In the $\phi=135^{\circ}$ case, the two tip vortices continue to approach each other in the $z$-direction during the observed time (figure $12 f$ ).

If the deceleration time is long like in the $\phi=135^{\circ}$ case, the bound vortex and side-edge vortex can start shedding towards the tip edge smoothly during deceleration. Moreover, the collision of two bound vortices in the gap between two plates and the consequent reconnection process are weakened. A large portion of the stopping tip vortices (bound vortices) can move downstream without being involved in the reconnection process, as exemplified for the $\phi=135^{\circ}$ and $A R=1$ case (figure 14). The stopping tip vortex does not merge with the starting tip vortex on the same side, but translates at a speed comparable to that of the starting tip vortex. Therefore, two 
(a)

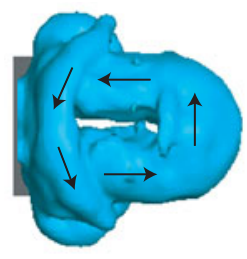

(b)

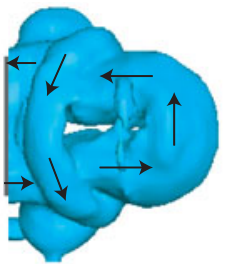

(c)

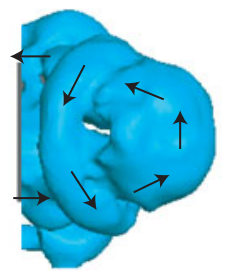

(d)

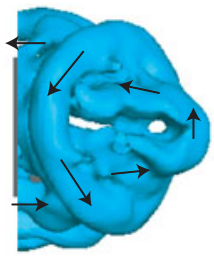

FIgURE 14. (Colour online) Vortex dynamics in the wake for the double plates with $\phi=135^{\circ}$ and $A R=1$ (back view from $-x,|\omega|=3$ ): (a) $\tau=0 ;(b) \tau=0.26$; (c) $\tau=0.79$; (d) $\tau=1.31$. Owing to a limited interrogation volume, only the positive $z$-side was observed.

separate inner vortex loops are formed in both positive and negative $z$-regions inside an outer vortex loop created by reconnection.

In this section, we have shown diverse morphodynamics of vortex structures in the wake and their dependence on stroke angle and aspect ratio. The morphodynamics presented here would be generally valid for scaled clapping models if a sinusoidal kinematics pattern (2.1) is preserved. However, if the Reynolds number is very low $(\sim 100)$ compared to that in this study (8400), the tip vortex separation from the rotating plate would not be as clear as in the current study; instead, the tip vortex core would be stretched following the rotating tip edge (Kim \& Gharib 2011). Therefore, the wake structure would have a different shape. The wake dynamics could also be affected by the angular velocity pattern of the plates. For example, for a large stroke angle such as $\phi=135^{\circ}$ with constant angular velocity but impulsive start and stop, the bound vortex would remain on the plate during the entire rotation instead of starting to shed towards the tip edge, which results in strong collision of two bound vortices between the two plates after the plates stop. For this reason, unlike the sinusoidal case shown in figure 14, it is expected that a single vortex loop will be formed in the wake without two separated inner vortex loops.

\section{Concluding remarks}

The vortex dynamics and thrust performance with a single plate and clapping double plates were investigated experimentally. The formation of a vortex loop composed of two counter-rotating tip vortices by reconnection is a distinct aspect of the clapping double plates, which does not appear in the single plate. Owing to the small gap between the two plates, the bound vortex cannot be shed into the wake, preserving its strength, but it interacts with the bound vortex on the other side. The cancellation of the two bound vortices and the reconnection of the two tip vortices result in the formation of a vortex loop. Depending on stroke angle and $A R$, the vortex loop can have diverse morphodynamics in the wake. For small stroke angles, the loop undergoes lateral compression and longitudinal elongation. In some cases, this deformation of the loop is followed by a switch of the major and minor axes.

The total impulse acting on the model is determined soon after the plate stops, regardless of the continuous deformation of the vortex in the wake. Thus, the vorticity distribution of the tip vortex formed when the plate stopped was examined to determine the effects of $A R$ on impulse for both single plate and double plates. As $A R$ of the plates decreases, the non-dimensional total impulse and instantaneous thrust coefficient increases over a broad range of stroke angles. The double plates generally produce a larger impulse per plate than the single plate with the same $A R$ and stroke 
angle. The advantage of the double plates in terms of thrust production becomes larger as $A R$ decreases. These results may give some insight into why low $A R$ wings of some insects, such as butterflies, can be beneficial aerodynamically. In slow forward flight modes or drag-based manoeuvring modes such as take-off and landing, low $A R$ wings may be more efficient than high $A R$ wings in that they can generate a higher thrust coefficient. Moreover, for clapping motions during pronation or supination, low $A R$ may be advantageous over high $A R$ since they can augment larger thrust by mutual interaction of the wing pair. Admittedly, it is necessary to explore more complicated kinematics and wing shapes in order to generalize this idea.

Even though several cases are explored in this study by varying $A R$ values and stroke angle, these cases do not exhaust all the possible scenarios of vortex formation in clapping models. This study was to explore the important dynamic mechanisms in thrust generation and vortex evolution in simple clapping models with emphasis on the dynamics of the tip vortex as well as its relation to thrust generation. From the understanding gained from this simple model, future work will focus on investigating the effect of the wing's aspect ratio on vortex structure and propulsive performance under various advance ratios by using a model to more realistically mimic the forward flight of flying animals.

\section{REFERENCES}

Betts, C. R. \& Wootton, R. J. 1988 Wing shape and flight behavior in butterflies (Lepidoptera, Papilionoidea and Hesperioidea) - a preliminary analysis. J. Expl Biol. 138, 271-288.

BRODSKY, A. K. 1991 Vortex formation in the tethered flight of the peacock butterfly Inachis io L. (Lepidoptera, Nymphalidae) and some aspects of insect flight evolution. J. Expl Biol. 161, 77-95.

Cooter, R. J. \& BAKer, P. S. 1977 Weis-Fogh clap and fling mechanism in Locusta. Nature 269, 53-54.

DudLey, R. 1990 Biomechanics of flight in neotropical butterflies - morphometrics and kinematics. J. Expl Biol. 150, 37-53.

Ellington, C. P. $1984 a$ The aerodynamics of hovering insect flight. 3. Kinematics. Phil. Trans. R. Soc. Lond. B 305, 41-78.

Ellington, C. P. $1984 b$ The aerodynamics of hovering insect flight. 4. Aerodynamic mechanisms. Phil. Trans. R. Soc. Lond. B 305, 79-113.

GaL, J. M. \& Blake, R. W. 1988 Biomechanics of frog swimming. 2. Mechanics of the limb-beat cycle in Hymenochirus boettgeri. J. Expl Biol. 138, 413-429.

Hussain, F. \& Husain, H. S. 1989 Elliptic jets. 1. Characteristics of unexcited and excited jets. J. Fluid Mech. 208, 257-320.

Johansson, L. C. \& LAUdeR, G. V. 2004 Hydrodynamics of surface swimming in leopard frogs (Rana pipiens). J. Expl Biol. 207, 3945-3958.

Kambe, T. \& TakaO, T. 1971 Motion of distorted vortex rings. J. Phys. Soc. Japan 31, 591-599.

KidA, S. \& TAKaOKA, M. 1994 Vortex reconnection. Annu. Rev. Fluid Mech. 26, 169-189.

KIM, D. \& GHARIB, M. 2011 Characteristics of vortex formation and thrust performance in drag-based paddling propulsion. J. Expl Biol. 214, 2283-2291.

MaXwORThy, T. 1979 Experiments on the Weis-Fogh mechanism of lift generation by insects in hovering flight. 1. Dynamics of the fling. J. Fluid Mech. 93, 47-63.

Melander, M. V. \& Hussain, F. 1989 Cross-linking of two antiparallel vortex tubes. Phys. Fluids A 1, 633-636.

Miller, L. A. \& Peskin, C. S. 2005 A computational fluid dynamics of 'clap and fling' in the smallest insects. J. Expl Biol. 208, 195-212.

Pereira, F. \& Gharib, M. 2002 Defocusing digital particle image velocimetry and the three-dimensional characterization of two-phase flows. Meas. Sci. Technol. 13, 683-694. 
Pereira, F., Stuer, H., Graff, E. C. \& Gharib, M. 2006 Two-frame 3D particle tracking. Meas. Sci. Technol. 17, 1680-1692.

Spedding, G. R. \& Maxworthy, T. 1986 The generation of circulation and lift in a rigid two-dimensional fling. J. Fluid Mech. 165, 247-272.

Stamhuis, E. J. \& Nauwelaerts, S. 2005 Propulsive force calculations in swimming frogs. II. Application of a vortex ring model to DPIV data. J. Expl Biol. 208, 1445-1451.

SUN, M. \& YU, X. 2003 Flows around two airfoils performing fling and subsequent translation and translation and subsequent clap. Acta Mechanica Sin. 19, 103-117.

WEIS-FOGH, T. 1973 Quick estimates of flight fitness in hovering animals, including novel mechanisms for lift production. J. Expl Biol. 59, 169-230.

Willert, C. \& GHARIB, M. 1992 Three-dimensional particle imaging with a single camera. Exp. Fluids 12, 353-358.

WU, J. C. 1981 Theory for aerodynamic force and moment in viscous flows. AIAA J. 19, 432-441. 